\title{
La scelta missionaria nell'attività del consiglio pastorale parrocchiale
}

\section{The Missionary Choice in the Activity of Parish Pastoral Council}

\begin{abstract}
The article discusses the missionary option as indicated by Pope Francis in his first apostolic exhortation. This challenging option can be lived fully only through a total commitment of all church structures. That is why it is interesting to reflect how one of the parish structures, namely the pastoral council, can contribute - according to its competences - to put into practice what has been enunciated in Evangelii gaudium.

A particular contribution of the parish pastoral council consists primarily in the development of an adequate parish pastoral plan, which would have as its main goal to educate the parish as a missionary community, responsible for the transmission of the Christian faith in today's world. The article also features some directions from the Papal document which must be taken into account and some practical suggestions which aid in reaching the delineated goals. A commitment of the parish pastoral council to fulfil this missionary choice enables this structure to see its role as oriented "to promote conformity of the life and actions of the People of God with the Gospel".
\end{abstract}

\section{Keywords}

Parish pastoral council, Pope Francis, Evangelii gaudium, evangelization, pastoral planning.

\section{Introduzione}

La prima esortazione apostolica di papa Francesco ha destato un grande interesse non solo nella Chiesa. Per la comunità dei discepoli del Signore non è tuttavia un documento che attiri una effimera e superficiale attenzione, ma costituisce un importante punto di riferimento ed aiuta a udire ed 
a capire „ciò che lo Spirito dice alle Chiese"lattraverso l'insegnamento del vescovo di Roma. Lui stesso ha sottolineato decisamente che quanto aveva espresso nell'Evangeliigaudium, ha un significato programmatico e dalle conseguenze importanti”2. Al centro di questo „programma” si trova „una conversione pastorale e missionaria" 3 di tutta la Chiesa come un requisito necessario per riprendere ,una nuova tappa evangelizzatrice, piena di fervore e dinamismo"'.

Nel presente articolo si cercherà di illustrare in quale modo la scelta missionaria si riferisce ad una delle strutture pastorali della Chiesa a livello parrocchiale, cioè al consiglio pastorale parrocchiale. Questo organismo di partecipazione e di corresponsabilità in seno alla comunità parrocchiale costituisce da qualche decennio uno degli strumenti che recano un prezioso aiuto „nel promuovere l'attività pastorale” ${ }^{\text {. Nel }} 2013$ nella Chiesa in Polonia ha avuto luogo il Primo Congresso Nazionale dei Consigli Pastorali Parrocchiali, il quale ha dato un nuovo e forte impulso alla loro crescita e maturazione ${ }^{6}$. La riflessione sulla conversione pastorale e missionaria nell'attività del consiglio pastorale si inserisce dunque nel processo di un ulteriore sviluppo di questa struttura nella prospettiva tracciata da papa Francesco.

Il tema sarà sviluppato in tre punti. Il primo presenterà il concetto della scelta missionaria esposta nell'Evangeliigaudium. Il secondo tratterà del consiglio pastorale nella prospettiva della scelta missionaria soprattutto sulla base dei documenti della Chiesa che fanno ricorso a quell'organismo. Al terzo punto si offriranno alcuni suggerimenti concreti perché il consiglio pastorale possa lavorare efficacemente secondo una chiave missionaria.

1 Atti degli Apostoli 2, 7.

2 Francesco, L'esortazione apostolica Evangelii gaudium sull'annunzio del Vangelo nel mondo attuale, 25 .

3 Evangelii gaudium, 25.

4 Evangelii gaudium, 17.

5 Codice di diritto canonico, $536 \S 1$.

6 Il Congresso è stato organizzato a Licheń (14-15 Settembre 2013) come una delle iniziative inserite nel triennale programma pastorale della Chiesa in Polonia: „La Chiesa: scuola e casa di communione" che aveva tra i suoi principali obiettivi il rinnovamento e rafforzamento delle strutture di comunione nelle comunità eccesiali: cf. K. Kantowski, S. Stułkowski: W komunii z Bogiem, in: Komisja Duszpasterstwa Konferencji Episkopatu Polski: W komunii z Bogiem. Kościót domem i szkoła komunii. Program duszpasterski Kościoła w Polsce na lata 2010-2013, S. Stułkowski (ed.), Poznań 2010, 16. 


\section{1. „Una scelta missionaria” secondo l'Evangelii gaudium}

La forte e decisiva spinta per una scelta missionaria da parte di papa Francesco deve essere vista nel contesto dei precedenti importanti interventi del Magistero della Chiesa nella seconda metà del secolo scorso e nei primi anni del nuovo Millennio legati ai temi dell'evangelizzazione. Non è qui il luogo per analizzarli dettagliatamente, comunque qualche accenno è indispensabile.

La Chiesa esiste per evangelizzare. Questo compito „,...) è la grazia e la vocazione propria della Chiesa, la sua identità più profonda"7. La Chiesa, nata dalla missione del Figlio e dalla missione dello Spirito Santo ${ }^{8}$, è stata inviata

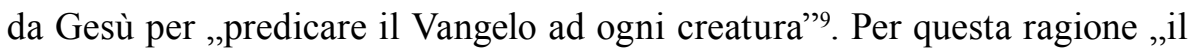
mandato di evangelizzare tutti gli uomini costituisce la missione essenziale della Chiesa"10. Questa attività al suo interno è l'unica missione, che assume però forme differenti, legate alle diverse circostanze del mondo in cui essa si svolge. Giovanni Paolo II, alludendo sia alle indicazioni del decreto concilare Ad gentes $^{11}$, che a quelle dell'Evangelii nuntiandi ${ }^{12}$, ha indicato tre situazioni: la prima concerne i popoli e i contesti in cui il Vangelo è sconosciuto oppure le comunità ecclesiali non sono ancora mature; poi, c'è la situazione di coloro che credono e vivono la propria fede con una responsabilità per la sua testimonianza negli ambienti della loro vita e con l'impegno missionario; la terza si attiene invece ai battezzati che hanno perso il senso della fede e si sono allontanati o addirittura hanno abbandonato non solo le pratiche della vita cristiana, ma la Chiesa stessa. A queste tre diverse situazioni corrispondono rispettivamente: la missione ad gentes, la cura pastorale e la nuova evangelizzazione ${ }^{13}$. Non può sfuggire il fatto che il Papa-Polacco avvertiva del pericolo, che nel contesto della missione globale della Chiesa, la missione ad gentes non fosse trascurata

7 Paolo VI, Esortazione apostolica Evangelii nuntiandi, 14.

8 Evangelii nuntiandi, 2.

9 Cf. Vangelo secondo Marco 16, 15.

${ }^{10}$ Evangelii nuntiandi, 14.

${ }^{11}$ Cf. Concilio Vaticano II, Decreto Ad gentes sull'attività missionaria della Chiesa, 6 .

${ }^{12} \mathrm{Nel}$ testo della Redemptoris missio dedicato a quelle tre situazioni non appare alcun esplicito richiamo all'Evangelii nuntiandi, comunque i nessi tra i due documenti in questa materia sono evidenti. Basta richiamare il numero 52 dell'esortazione di Paolo VI.

${ }^{13}$ Giovanni Paolo II, Lettera enciclica Redemptoris missio circa la permanente validità del mandato missionario, 33 . 
o dimenticata ${ }^{14}$. Notando inoltre che non è possibile stabilire i confini fra questi tre tipi di evangelizzazione, sottolineava decisamente che „senza la missione ad gentes la stessa dimensione missionaria della Chiesa sarebbe priva del suo significato fondamentale e della sua attuazione esemplare"15. Anche nell'altro importante documento, dato alla Chiesa all'inizio del nuovo Millennio, Giovanni Paolo II si è rivolto a tutti i credenti con un forte appello: „(...) occorre riaccendere in noi lo slancio delle origini, lasciandoci pervadere dall'ardore della predicazione apostolica seguita alla Pentecoste. (...) Questa passione non mancherà di suscitare nella Chiesa una nuova missionarietà (...) che dovrà coinvolgere la responsabilità di tutti i membri del Popolo di Dio"16.

Similmente, papa Benedetto XVI ha messo in evidenza la necessità del nuovo ,slancio missionario (...) di promuovere una nuova evangelizzazione" 17 . Convocato da lui il Sinodo dei Vescovi dedicato al tema della nuova evangelizzazione per la trasmissione della fede cristiana, indicava nell'Instrumentum laboris il bisogno della „nuova vitalità” e la necessità che la Chiesa „recuperi energie, volontà, freschezza e ingegno nel suo modo di vivere la fede e di trasmetterla"18. È stato anche affermato che "sottolineare che in questo momento della storia la Chiesa è chiamata a compiere una nuova evangelizzazione, vuol dire intensificare l'azione missionaria per corrispondere pienamente al mandato del Signore"19.

Con la sua esortazione apostolica papa Francesco non solo ribadisce e sottolinea quelle linee dell'insegnamento dei suoi predecessori, ma richiede con decisione da tutta la Chiesa una „trasformazione missionaria" ${ }^{20}$. Lo fa, perché è consapevole e convinto che l'azione missionaria costituisca,il paradigma di ogni opera della Chiesa" ${ }^{21}$. Questa argomentazione assomiglia molto a quello che scriveva Giovanni Paolo II affermando che la missione ad

\footnotetext{
${ }^{14}$ Redemptoris missio, 34 .

${ }^{15}$ Redemptoris missio, 34.

${ }^{16}$ Giovanni Paolo II, Lettera apostolica Novo millennio ineunte al termine del Grande Giubilleo dell'Anno Duemila, 40.

${ }^{17}$ Benedetto XVI, Lettera apostolica in forma di „Motu Proprio” Ubicumque et semper, con la quale si istituisce il Pontificio Consiglio per la Promozione della Nuova Evangelizzazione.

${ }^{18}$ Sinodo dei Vescovi (2012), XIII Assemblea generale ordinaria, La nuova evangelizzazione per la trasmissione della fede cristiana, Instrumentum laboris (2012), 49.

${ }^{19}$ Sinodo dei Vescovi (2012), Instrumentum laboris, 88.

${ }^{20}$ È questo il titolo del capitolo primo dell'Evangelii gaudium.

${ }^{21}$ Evangelii gaudium, 15.
} 
gentessi rivela come,,un'attuazione esemplare" per tutta l'attività della Chiesa. $\mathrm{Nel}$ paradigma missionario richiamato da Francesco si trova innanzitutto il dinamismo di ,uscita" 22 che caratterizzava la missione di Gesù e della Chiesa primitiva. Anche oggi la Chiesa è chiamata ad essere una comunità missionaria piena del simile dinamismo che si esprime nel prendere l'iniziativa, nel coinvolgimento, nell'accompagnamento, nel portare frutti e nel festegiare ${ }^{23}$.

La trasformazione missionaria della Chiesa e legato ad essa il dinamismo di „uscita” richiedono la necessità di lasciare le proprie abitudini, comodità e avere il coraggio di andare verso quello che è sconosciuto ${ }^{24}$. Questo non è facile. Per questa ragione papa Bergoglio esprime il suo desiderio e la speranza che la Chiesa riprenda la strada di ,una conversione pastorale e missionaria" ${ }^{25}$. Tale strada consiste sopratutto nel prendere coscienza che „non si può lasciare le cose come stanno”, e che oggi alla Chiesa non serve „una semplice amministrazione”, ma essa deve essere in tutte le regioni ,in uno stato permanente della missione" ${ }^{26}$. Sembra che Francesco attinga qui da Lineamenta del Sinodo dei Vescovi del 2012. In quel documento è stato affermato: „Nuova evangelizzazione è allora sinonimo di missione; chiede la capacità di ripartire, di oltrepassare i confini, di allargare gli orizzonti. La nuova evangelizzazione è il contrario dell'autosufficienza e del ripiegamento su sé stessi, della mentalità dello status quo e di una concezione pastorale che ritiene sufficiente continuare a fare come si è sempre fatto. Oggi il «business as usual non basta più»" ${ }^{27}$.

Chi nella Chiesa ha capito in che cosa consista la conversione pastorale e missionaria, è capace di agire in base a una scelta missionaria, la quale può trasformare tutto al servizio dell'opera d'evangelizzazione. Secondo il papa: „le consuetudini, gli stili, gli orari, il linguaggio e ogni struttura ecclesiale”

${ }^{22}$ Evangelii gaudium, 20.

${ }^{23}$ Evangelii gaudium, 24.

${ }^{24}$ Evangeli igaudium, 20.

${ }^{25}$ Evangeli gaudium, 25. Nell'Instrumentum laboris si legge: „La nuova evangelizzazione impegna tutti i soggetti ecclesiali (singoli, comunità, parrocchie, diocesi, Conferenze Episcopali, movimenti, gruppi ed altre realtà ecclesiali, religiosi e persone consacrate) ad una verifica della vita ecclesiale e dell'azione pastorale, assumendo come punto di analisi la qualità della propria vita di fede, e la sua capacità di essere strumento di annuncio, secondo il Vangelo": Sinodo dei Vescovi (2012), Instrumentum laboris, 77.

${ }^{26}$ Evangelii gaudium, 25.

${ }^{27}$ Sinodo dei Vescovi (2012), XIII Assemblea generale ordinaria, La nuova evangelizzazione per la trasmissione della fede cristiana, Lineamenta (2011), 10. 
devono diventare „un canale per l'evangelizzazione del mondo attuale, più che per l'autopreservazione" 28 .

La trasformazione missionaria e la scelta missionaria si inseriscono nella riforma della Chiesa. Francesco la intende secondo quello che hanno affermato i Padri del Concilio Vaticano II: „ogni rinnovamento della Chiesa consiste essenzialmente in un'accresciuta fedeltà alla sua vocazione"29. Questa non è altro che riprendere continuamente l'impegno di evangelizzare attraverso il quale la Chiesa cerca di offrire a tutti quello che ha come un tesoro più grande: Gesù Cristo che vuole offrire a tutti la sua vita divina. Per poter portarLo a tutti, la Chiesa - come il Papa mette in evidenza - non deve avere paura di uscire per le strade anche se quello la farà accidentata, ferita e sporca. Tale uscita implica che essa non può rimanere più chiusa in sé stessa, aggrappata alle proprie sicurezze e comodità. Non può rinchiudersi nelle strutture che le danno una falsa protezione e nelle abitudini in cui si sente tranquilla, ma con nuovo vigore e nuova freschezza deve uscire verso tutti quelli che ,vivono senza la forza, la luce e la consolazione dell'amicizia con Gesù Cristo, senza una comunità di fede che li accolga, senza un orizzonte di senso e di vita" ${ }^{30}$.

La sfida che sta di fronte alla Chiesa nella prospettiva della sua trasformazione missionaria significa che la comunità dei discepoli di Gesù, e in modo speciale quelli che sono impegnati particolarmente nel lavoro pastorale, devono confrontarsi e superare le varie tentazioni che almeno in una parte provengono dalla cultura attuale globalizzata. Nella vita dei sacerdoti e delle persone consacrate si osserva la mancanza di una vera passione per l'evangelizzazione. L'impegno ecclesiale viene trattato come un lavoro separato dalla propria identità. Si cerca piuttosto come finirlo al più presto possibile per godersi del tempo libero. Un benessere personale e i desideri di essere „grande” e ,primo” secondo i criteri del mondo diventano più importanti della gloria di Dio. Di fronte alle molte difficoltà spuntano scoraggiamento e sfiducia, non c'è più posto per un vero dinamismo e una naturale gioia proveniente dalla partecipazione all'opera di Dio stesso e dalla

${ }^{28}$ Evangelii gaudium, 27.

${ }^{29}$ Evangelii gaudium, 26. Il Papa cita qui il numero 6 del Decreto Ad gentes del Concilio Vaticano II. Al numero 77 dell'Instrumentum laboris del Sinodo dei Vescovi del 2012 si legge: „La nuova evangelizzazione impegna tutti i soggetti ecclesiali (...) ad una verifica della vita ecclesiale e dell'azione pastorale, assumendo come punto di analisi la qualità della propria vita di fede, e la sua capacità di essere strumento di annuncio, secondo il Vangelo".

30 Evangelii gaudium, 49. 
condivisione con gli altri del tesoro della fede. Quando manca la fiducia prevale l'atteggiamento della difesa e della conservazione di quello che sembra ancora funzionare. Si continua una pastorale delle apparenze come se tutto andasse bene. Al posto dell'evangelizzazione appare la critica degli altri, oppure una concentrazione sui meccanismi di controllo. Si osserva un esagerato attaccamento agli aspetti organizzativi e alle azioni che si ripete senza riflettere se esse rechino ancora qualcosa di significativo alla vita delle genti. Succede che la pastorale è spersonalizzata, priva del contatto reale con la gente ${ }^{31}$. Per superare impropri atteggiamenti e varie tentazioni occorre continuamente ritornare allo spirito della nuova evangelizzazione, che è sempre „un'evangelizzazione con Spirito Santo"32.

\section{II consiglio pastorale parrocchiale nella prospettiva della scelta missionaria}

L'impegno della trasformazione missionaria riguarda tutti i soggetti ecclesiali. Tra essi un ruolo particolare spetta alla parrocchia, la quale è chiamata, attraverso tutte le sue attività, a incoraggiare e formare i suoi membri perché siano agenti dell'evangelizzazione. Il rinnovamento delle parrocchie deve orientarle completamente verso la missione, edificarle come ambiti di comunione viva e di partecipazione, farle più vicine alla gente ${ }^{33}$. Per poter realizzare tali obiettivi è necessario l'impegno di tutti coloro che la formano. Anche il consiglio pastorale parrocchiale, come un soggetto attivo della pastorale, è chiamato a offire il proprio contributo. In questa parte si cercherà di trarre da alcuni documenti della Chiesa che parlano, oppure alludono esplicitamente al consiglio pastorale parrocchiale, ciò che riguarda la sua dimensione missionaria ed evangelizzatrice.

Al primo posto occorre rilevare le indicazioni provenienti dal motu proprio Ecclesiae sanctae pubblicato da Paolo VI nel $1966^{34}$. Il documento stabilisce che „è compito del consiglio pastorale studiare, esaminare tutto ciò che

${ }^{31}$ Evangelii gaudium, 78-101.

32 Evangelii gaudium, 261.

${ }^{33}$ Evangelii gaudium, 28.

${ }^{34}$ È un documento in cui si trovano tra l'altro le norme relative al consiglio pastorale diocesano, le quali si possono riferire - mutatis mutandis - al consiglio pastorale parrocchiale. 
concerne le attività pastorali, e proporre quindi conclusioni pratiche, al fine di promuovere la conformità della vita e dell'azione del popolo di Dio con il Vangelo" 35 . Abbiamo qui a che fare con una norma molto significativa. Essa offre una prospettiva giusta e profonda nella quale si vedono tutti i compiti del consiglio pastorale. Che cosa significa „la conformità della vita e dell'azione del popolo di Dio con il Vangelo"? Significa fra l'altro ritornare continuamente a quello che dice il Vangelo ${ }^{36}$. E il suo messaggio è molto chiaro: la Chiesa è chiamata a "fare i discepoli”, a ,predicare il Vangelo ad ogni creatura" ${ }^{37}$. La Chiesa è una comunità missionaria. L'espressione ,promuovere la conformità della vita e dell'azione del popolo di Dio con il Vangelo" permette di capire che il consiglio pastorale è il luogo della consultazione ecclesiale per una verifica del dinamismo evangelico e missionario della comunità parrocchiale ${ }^{38}$.

L'importanza del testo sopra citato viene confermata nella lettera circolare Omnes christifideles ${ }^{39}$, emanata dalla Congregazione per il Clero nel 1973. Nel brano dedicato ai compiti affidati ai consigli pastorali è espressamente richiamato il testo di Ecclesiae sanctae. Se si ricorda che l'Omnes christifideles costituisce fino ad oggi l'unico testo ufficiale della Chiesa dedicato integralmente ai consigli pastorali, la presenza in esso di quel compito è più che significativa. Dispiace che i documenti posteriori, e specialmente il Codice di diritto canonico, non si siano serviti dell'espressione preferita sia nell'Ecclesiae sanctae che nell'Omnes christifideles. La lettera Omnes christifideles fornisce inoltre un elenco più dettagliato dei compiti affidati al consiglio pastorale. Tra essi vanno annoverati tra l'altro: le proposte e i suggerimenti relativi alle iniziative missionarie, catechetiche e apostoliche ${ }^{40}$. Anche se le iniziative missionarie bisogna intenderle qui in senso stretto, cioè come le attività

${ }^{35}$ Paolo VI, Motu proprio Ecclesiae sanctae circa l'applicazione dei decreti conciliari Christus Dominus, Presbyterorum ordinis, Ad gentes, I, 16 § 1.

${ }^{36}$ Quando la Chiesa cerca „di tornare alla fonte e recuperare la freschezza originale del Vangelo spuntano nuove strade, metodi creativi, altre forme di espressione, segni più eloquenti, parole cariche di rinnovato significato per il mondo attuale": Evangelii gaudium, 11.

37 Vangelo secondo Marco 16, 15.

38 A. Borras, La parrocchia. Diritto canonico e prospettive pastorali, Bologna 1997, 222.

${ }^{39}$ Congregazione per il Clero, Lettera circolare Omnes christifideles sui consigli pastorali, 9. Il documento è dedicato ai consigli pastorali diocesani. Al numero 12 è stato comunque affermato che ,niente impedisce che nell'ambito della diocesi siano istituiti consigli della stessa natura e della stessa funzione, tanto parrocchiali che zonali’”.

${ }^{40}$ Omnes christifideles, 9. 
riguardanti la missione ad gentes, niente impedisce che oggi esse siano viste nel senso più ampio indicato da papa Francesco.

Un'altro testo che merita di essere richiamato a proposito del tema analizzato, è quello di Giovanni Paolo II, contenuto nell'esortazione apostolica Christifideles laici del 1988. Nella parte dedicata all'impegno apostolico dei laici nella parrocchia, il Papa cita il brano del decreto conciliare Apostolicam actuositatem, in cui si esortano i fedeli laici affinché espongano alla comunità della Chiesa i problemi propri e del mondo e le questioni che riguardano la salvezza del mondo, perché siano esaminati e risolti con il concorso di tutti ${ }^{41}$. Secondo il Papa, quell'accenno conciliare „deve trovare il suo adeguato e strutturato sviluppo nella valorizzazione più convinta, ampia e decisa dei consigli pastorali parrocchiali" ${ }^{2}$. Nell'ambito dei vari compiti del consiglio appare dunque con evidenza quello che riguarda una comune riflessione dei suoi membri sulla salvezza del mondo. Tale compito deve allora significare un forte addentramento nella questione dell'evangelizzazione nella comunità parrocchiale. La dimensione missionaria nei vari suoi elementi si rivela come una realtà dalla quale non si può prescindere nell'attività del consiglio pastorale. Tale affermazione trova la sua conferma nell'enunciazione che segue immediatamente la richiesta della valorizzazione dei consigli pastorali parrocchiali. Giovanni Paolo II nota: „Nelle circostanze attuali i fedeli laici possono e devono fare moltissimo per la crescita di un'autentica comunione ecclesiale all'interno delle loro parrocchie e per ridestare lo slancio missionario verso i non credenti e verso gli stessi credenti che hanno abbandonato o affievolito la pratica della vita cristiana" ${ }^{\text {"43 }}$. E un testo che oggi, come pure in quel tempo in cui l'esortazione apostolica è stata pubblicata, esige una forte ripresa. Esso non riguarda ovviamente soltanto i membri del consiglio pastorale parrocchiale, ma tutti i fedeli laici che compongono la parrocchia, e in modo particolare quelli che per vari motivi vi sono coinvolti di più. Il consiglio pastorale può trarne un compito di grande importanza per il suo funzionamento e per le sue priorità nel contesto odierno segnato dalla vasta secolarizzazione e dall'indifferenza religiosa.

${ }^{41}$ Giovanni Paolo II, Esortazione apostolica Christifideles laici sulla vocazione e missione dei laici nella Chiesa e nel mondo, 27.

${ }^{42}$ Christifideles laici, 27.

${ }^{43}$ Christifideles laici, 27. 
Anche l'esortazione Evangelii gaudium contiene alcune osservazioni che si possono riferire all'attività dei consigli pastorali parrocchiali. Li troviamo innanzitutto nel capitolo dedicato al tema della pastorale nella conversione. Prima, papa Francesco si sofferma in modo generale sulle strutture ecclesiali, senza indicarne di concrete, e offre a proposito due osservazioni. La prima fornisce un criterio per giudicare la loro utilità. Le strutture „servono quando c'è una vita che le anima, le sostiene e le giudica. Senza vita nuova e autentico spirito evangelico, senza «fedeltà della Chiesa alla propria vocazione», qualsiasi nuova struttura si corrompe in poco tempo" ${ }^{\prime 4}$. In altre parole: le strutture ecclesiali non possono bloccare o limitare il dinamismo evangelizzatore, ma devono favorirlo. La seconda osservazione spiega come si deve intendere la riforma delle strutture. Anche qui il criterio è legato unicamente alla scelta missionaria. Secondo il Papa, occorre „fare in modo che esse diventino tutte più missionarie, che la pastorale orddinaria in tutte le sue istanze sia più espansiva e aperta, che ponga gli agenti pastorali in costante atteggiamento di «uscita» e favorisca così la risposta positiva di tutti coloro ai quali Gesù offre la sua amicizia" ${ }^{\text {". }}$. Il funzionamento dei consigli pastorali parrocchiali e il loro rinnovamento devono essere visti alla luce di queste due indicazioni.

Una particolare attenzione merita inoltre la riflessione contenuta nel punto 31 dello stesso capitolo, dove si tratta del vescovo e della sua responsabilità per favorire una comunione missionaria nella sua Chiesa particolare. In questo impegno il vescovo deve ,stimolare e ricercare la maturazione degli organismi di partecipazione proposti dal Codice di diritto canonico" ${ }^{46}$. L'Evangelii gaudium fa qui riferimento alle varie strutture di partecipazione in segno alla comunità diocesana e alle comunità parrocchiali. Nella nota viene tra l'altro richiamato il canone $536 \mathrm{del}$ Codice, il quale è dedicato al consiglio pastorale parrocchiale ${ }^{47}$. Avvalendosi delle strutture di partecipazione il

${ }^{44}$ Evangelii gaudium, 26. Papa Benedetto XVI, durante la sua visita apostolica in Germania, ha affermato: „In Germania la Chiesa è organizzata in modo ottimo. Ma, dietro le strutture, vi si trova anche la relativa forza spirituale, la forza della fede nel Dio vivente? Sinceramente dobbiamo però dire che c'è un'eccedenza delle strutture rispetto allo Spirito. (...) La vera crisi della Chiesa nel mondo occidentale è una crisi di fede. Se non arriveremo ad un vero rinnovamento nella fede, tutta la riforma strutturale resterà inefficace": Benedetto XVI, Discorso all'incontro con il Consiglio del Comitato Centrale dei Cattolici Tedeschi (ZDK), 24 settembre 2011.

${ }^{45}$ Evangelii gaudium, 27.

${ }^{46}$ Evangelii gaudium, 31.

${ }^{47}$ Evangelii gaudium. Vedi la nota 34. 
vescovo è chiamato ad, ascoltare tutti, e non solo alcuni, sempre pronti a fargli complimenti”"48. Il Papa ricorda inoltre che „'obiettivo di questi processi partecipativi non sarà principalmente l'organizzazione ecclesiale, bensì il sogno missionario di arrivare a tutti" ${ }^{\prime 4}$. Il conisglio pastorale parrocchiale trova qui un compito molto chiaro. Tutta la sua attività deve essere vista nell'ottica missisonaria con una particolare attenzione verso quelli che si sono allontanati, che hanno abbandonato la Chiesa, oppure non sono mai stati suoi membri.

Alla fine di questa parte è necessario prestare attenzione a quello che papa Bergoglio ha sottolineato nell'ultimo punto del capitolo sulla pastorale nella conversione. La ripresa della pastorale in chiave missionaria non consiste nel fare una cosmetica di quello che si fa. Bisogna mettere in questione o addirittura abbandonare il criterio pastorale del „si è fatto sempre cosi”, e ripensare, con audacia e creatività, gli obiettivi, le strutture, lo stile e i metodi evangelizzatori nella comunità ${ }^{50}$. Francesco avverte tuttavia che non basta una semplice individuazione dei nuovi fini. Perchè quel lavoro non sia destinato a restare una mera teoria o un sogno, ci vuole una ricerca comunitaria dei mezzi che permetteranno di raggiungere gli scopi tracciati. Un ambito adeguato per condurre tale ricerca comunitaria nella parrocchia è indubbiamente il consiglio pastorale parrocchiale con il concorso di tutti e sotto la guida del parroco.

\section{Alcune indicazioni pratiche per il consiglio pastorale parrocchiale alla luce dell'Evangelii gaudium}

Nella parte precedente si è cercato di offrire alcuni suggerimenti generali provenienti dai documenti della Chiesa che hanno consentito di vedere l'attività del consiglio pastorale parrocchiale nella prospettiva della scelta missionaria alla quale la Chiesa viene oggi particolarmente chiamata. Ora, alla luce dell'Evangelii gaudium, saranno fornite indicazioni più dettagliate su come può lavorare il consiglio pastorale in chiave missionaria.

\footnotetext{
${ }^{48}$ Evangelii gaudium, 31 .

${ }^{49}$ Evangelii gaudium, 31 .

${ }^{50}$ Evangelii gaudium, 33.
} 
Prima che il consiglio cominci qualsiasi attività in questa direzione, è doveroso che esamini la presenza dello spirito missionario al suo interno. Si tratta semplicemente che le persone che compongono il consiglio pastorale siano permeate di un tale spirito. La scelta missionaria può essere infatti realizzata dalle persone che vivono la propria fede come un grande dono ricevuto gratuitamente e che hanno una viva coscienza che quel dono deve essere necessariamente condiviso con gli altri ${ }^{51}$. Dove manca lo spirito missionario, domina lo stile di conservazione, prevalgono ripetizione e continuazione delle stesse iniziative, perché non si vede il bisogno di cercare quelli che non hanno ancora scoperto la bellezza e la gioia della fede oppure non ritengono più il Vangelo come qualcosa di veramente significativo per la loro vita. Dove invece i membri del consiglio hanno l'entusiasmo della fede, che li spinge e li apre verso la sfida dell'indifferenza religiosa, dove non si accontentano dello stato presente, la loro attività nel consiglio tenderà sempre a riflettere su come comunicare la Buona Novella agli altri. Anche se gli statuti dei consigli pastorali parrocchiali non esigono direttamente che i suoi membri siano caratterizzati dallo spirito missionario, tale atteggiamento è necessario nella situazione attuale, affinché il consiglio sia uno strumento adeguato per l'evangelizzazione del mondo attuale ${ }^{52}$.

I membri del consiglio pastorale sono chiamati a prestare il loro aiuto nel ,promuovere l'attività pastorale" ${ }^{53}$. Uno degli strumenti per realizzare questo compito generale è - indicata da molti statuti o regolamenti di quell'organismo - l'elaborazione del progetto pastorale parrocchiale. Tale progetto tende ad attuare per la concreta comunità le linee e gli orientamenti della Chiesa universale, nonché del piano pastorale nazionale e diocesano. La portata di questo compito è più che ovvia. Grazie all'elaborazione del progetto pastorale per una determinata comunità, tutti hanno la possibilità di notare che a livello parrocchiale si cerca di fare quello che fa tutta la Chiesa. D'altra parte, la nuova evangelizzazione con rinnovato slancio missionario non si realizzerà mai se non sarà ripresa dalle comunità diocesane e parrocchiali. L'importanza del progetto pastorale parrocchiale è legata anche al fatto che esso permette

51 „La fede (...) cresce quando è vissuta come esperienza di un amore ricevuto e quando viene comunicata come esperienza di grazia e di gioia": Benedetto XVI, Lettera apostolica in forma di motu proprio Porta fidei con la quale si indice L'Anno della Fede, 7.

${ }^{52}$ Cf. J. Gręźlikowski, Rady parafialne w świetle aktualnych potrzeb Kościoła w Polsce, „Homo Dei” 270 (2004/1), 55.

${ }^{53}$ Codice di diritto canonico, $536 \S 1$. 
di solito di realizzare altri compiti dettagliati del consiglio. Nel progetto pastorale parrocchiale vengono individuati gli obiettivi concreti a medio e a lungo termine, nonché le condizioni di pertinenza teologica e operativa dei medesimi. Elaborare il progetto pastorale parrocchiale significa in realtà determinare quale modello della Chiesa la comunità parrocchiale vuole e deve edificare, quali obiettivi deve realizzare, verso quale futuro muoversi. Il progetto pastorale parrocchiale affida poi al programma pastorale, che viene preparato e realizzato nella parrocchia per ogni anno, la determinazione degli obiettivi a breve termine, dei mezzi, dei percorsi e delle persone ${ }^{54}$.

L'elaborazione del progetto pastorale parrocchiale per il tempo e la situazione presente trova nell'Evangelii gaudium l'imprescindibile punto di riferimento. Ai membri del consiglio sono stati forniti i principali e chiari orientamenti per la sua preparazione. Il progetto pastorale deve essere così costruito, attraverso una retta determinazione di obiettivi, mezzi, percorsi e iniziative, affinché tutto sia al servizio dell'opera d'evangelizzazione. Si tratta in realtà che il progetto pastorale tenda a costruire un modello della parrocchia, il quale la renda una comunità missionaria piena del rinnovato dinamismo evangelizzatore verso tutti quelli che si sono allontanati dalla vita ecclesiale, che sono indifferenti, che hanno perso la fede, ma anche verso coloro che credono, perché la loro fede cresca e si rafforzi.

In tale prospettiva si può parlare di una ,trasformazione missionaria” dello stesso progetto pastorale parrocchiale. Tra gli obiettivi da raggiungere nel progetto occorre annoverare i seguenti: orientare completamente la parrocchia verso la missione ${ }^{55}$, coinvolgere tutti nell'opera di evangelizzazione ${ }^{56}$, arrivare a tutti, senza eccezioni, con il Vangelo per inserirlo nella loro vita come Buona Novella, come una proposta valida, significativa e vivificante; farsì che la parrocchia sia una comunità aperta e accogliente, una comunità di comunità $^{57}$. Questi obiettivi sono naturalmente a lungo termine, ma non privi di concretezza. Tra le condizioni che condurranno a questi obiettivi si possono indicare: aiutare le famiglie perché abbiano un ruolo sempre più attivo nel

${ }^{54}$ S. Lanza, Progetto, discernimento, verifica pastorale, in: Aa. Vv., Creatività dello Spirito Santo e programmazione pastorale, Roma 1998, 78.

${ }^{55}$ Evangelii gaudium, 28.

${ }^{56}$ Papa Francesco presta attenzione al fatto che nella Chiesa si cessi di parlare separatamente dei credenti come „discepoli” e come „missionari”, ma che tutti si risconoscano come „discepolimissionari": Evangelii gaudium, 120.

${ }^{57}$ Evangelii gaudium, 28; 46-47; 49. 
processo di trasmissione della fede ${ }^{58}$, privilegiare i poveri e i malati, perché proprio loro - secondo quello che faceva e diceva Gesù - sono i destinatari privilegiati dell'annunzio evangelico ${ }^{59}$, promuovere la pietà popolare ${ }^{60}$, dare il maggiore peso alle comunità di base e ai movimenti impegnati nell'opera evangelizzatrice, specialmente quelli che si caratterizzano per il forte fervore evangelizzatore e per una capacità di dialogo con il mondo ${ }^{61}$; riprendere una vera formazione dei parrocchiani, perché siano agenti dell'evangelizzazione ${ }^{62}$. Da rilevare sono anche i mezzi adeguati agli obiettivi tracciati. Attingendo dalle riflessioni di papa Francesco occorre sottolineare fra l'altro: il ruolo centrale del primo annuncio (kerygma) a ogni livello della catechesi, l'inserimento dell'iniziazione mistagogica ${ }^{63}$, l'introduzione dell'accompagnamento segnato dalla prossimità e dal rispetto, il quale condurrà allo stesso tempo alla vera libertà e al coraggio di maturare nella vita cristiana ${ }^{64}$, „uno studio serio e perseverante della Bibbia, come pure (...) la sua lettura orante personale e comunitaria" ${ }^{65}$, il dialogo e la testimonianza personale ${ }^{66}$.

A proposito dei mezzi adeguati per raggiungere i fini individuati, occorre indicare l'insistenza del papa sull'impiego della ricerca comunitaria. Quando essa manca, tutto „è condannato a tradursi in mera fantasia"67. Il consiglio pastorale parrocchiale costituisce uno degli strumenti per tale ricerca. Questo riguarda non solo l'apporto che esso - come un organismo unitario - reca attraverso la realizzazione dei suoi compiti propri, ma si riferisce altresì al suo interno, cioè, a certi principi che determinano le regole del suo funzionamento

${ }^{58}$ Sinodo dei Vescovi (2012), Instrumentum laboris, 111. Questo documento richiama le risposte che sono arrivate alla Segreteria del Sinodo, le quali informavano della "costituzione di gruppi di famiglie (...) animati dalla fede cristiana che ha permesso a tanti coniugi di affrontare meglio le difficoltà a cui sono andati incontro, dando così anche una testimonianza chiara della fede cristiana": Instrumentum laboris, 112.

${ }^{59}$ Vangelo secondo Luca 4, 18.

${ }^{60}$ Evangelii gaudium, 123-126.

${ }^{61}$ Evangelii gaudium, 29; 130-131.

${ }^{62}$ Evangelii gaudium, 29; 121.

${ }^{63}$ L'iniziazione mistagogica „significa essenzialmente due cose: la necessaria progressività dell'esperienza formativa in cui interviene tutta la comunità ed una rinnovata valorizzazione dei segni liturgici dell'iniziazione cristiana: Evangelii gaudium, 166.

${ }^{64}$ Evangelii gaudium, 163-166; 169-173.

${ }^{65}$ Evangelii gaudium, 175.

${ }^{66}$ Evangelii gaudium, 127-129.

${ }^{67}$ Evangelii gaudium, 33. 
interno. Questo vuol dire semplicemente che il parroco e i membri del consiglio non possono concentrarsi su sé stessi, né limitare quella ricerca alle opinioni e idee di alcuni cosidetti ,eletti” che sanno tutto. All'interno del consiglio deve svolgersi una ricerca comunitaria che rispetti e valorizzi gli apporti di ciascuno. Vale qui la pena di richiamare quello che scriveva Giovanni Paolo II a proposito dei consigli pastorali e presbiterali: „Significativo ciò che san Benedetto ricorda all'Abate del monastero, nell'invitarlo a consultare anche i più giovani: «Spesso ad uno più giovane il Signore ispira un parere migliore ». E san Paolino di Nola esorta: «Pendiamo dalla bocca di tutti i fedeli, perché in ogni fedele soffia lo Spirito di Dio»" ${ }^{\natural 6}$.

Dalle numerose indicazioni contenute nell'Evangelii gaudium, merita anche di prendere in considerazione e impiegare per l'attività del consiglio pastorale parrocchiale, l'appello del papa, perché nella Chiesa non ci sia un esagerato concentramento su discussioni oppure su elementi organizzativi, ma prevalga un vero coinvolgimento nei lavori e l'autentico spirito di servizio che il consiglio offre attraverso la realizzazione dei suoi compiti. Esso è un organismo di studio, di valutazione e di proposta, però i suoi membri non possono mai sentirsi come gli esperti esterni che offrono agli altri le istruzioni elaborate, ma devono essere permeati dal fervore evangelico che permette loro di cogliere i vari problemi con la coscienza viva che anche loro sono responsabili della missione evangelizzatrice ${ }^{69}$.

\section{Conclusione}

La sfida della trasformazione missionaria che papa Francesco pone davanti alla Chiesa esige l'impegno di tutti i soggetti ecclesiali. Il nuovo slancio missionario non è pensabile come l'opera di alcuni più permeati dallo spirito evangelico o elargiti con i carismi adeguati. Si tratta di uno sforzo di tutta la Chiesa. Per questa ragione è stato opportuno analizzare come il consiglio pastorale parrocchiale, in quanto uno dei soggetti attivi della pastorale in parrocchia, possa impegnarsi e recare il proprio apporto - secondo le sue competenze - nella realizzazione di quello che è stato esposto nell'Evangelii gaudium.

\footnotetext{
${ }^{68}$ Novo millennio ineunte, 45.

${ }^{69}$ Evangelii gaudium, 95-97.
} 
È stato rilevato che il contributo del consiglio pastorale si evidenzierà soprattutto nell'elaborazione di un adeguato progetto pastorale parrocchiale, il quale porrà come uno dei suoi obiettivi principali l'edificazione della parrocchia come una comunità missionaria - responsabile della trasmissione della fede. Il progetto pastorale, e poi il più dettagliato programma pastorale per ogni anno, danno la possibilità di mettere in pratica le numerose indicazioni concrete contenute nel documento papale. L'elaborazione del progetto e dei programmi pastorali richiede una vera ricerca comunitaria che coinvolga tutti i membri del consiglio.

Riferendo le riflessioni contenute nell'Evangelii gaudium all'attività del consiglio pastorale parrocchiale si può concludere che papa Francesco vorrebbe che questa struttura ecclesiale realizzasse i compiti che le sono stati delineati nella lettera circolare Omnes christifideles, cioè: „studiare, esaminare tutto ciò che concerne le attività pastorali e proporre quindi conclusioni pratiche, al fine di promuovere la conformità della vita e dell'azione del popolo di Dio con il Vangelo".

\section{Bibliography}

Paul VI, Apostolic exhortation Evangelii nuntiandi, 08.12.1975, http:/www.vatican.va/ holy_father/paul_vi/apost_exhortations/documents/hf_p-vi_exh_19751208_evangeliinuntiandi_en.html (30.05.̄̄014).

John Paul II, Encyclical letter Redemptoris missio on the permanent validity of the Church's missionary mandate, 07.12.1990, http://www.vatican.va/holy_father/john paul_ii/encyclicals/documents/hf_jp-ii_enc_07121990_redemptoris-missio_en.html (30.05.2014).

Francis, Apostolic exhortation Evangelii gaudium on the proclamation of the Gospel in today's world, 24.11.2013, http://w2.vatican.va/content/francesco/en/apost exhortations/documents/papa-francesco_esortazione-ap_20131124_evangelii-gaudium. html (30.05.2014).

Synod of Bishops, XIII Ordinary General Assembly, The new evangelization for the transmission of the christian faith, Lineamenta (2011), http://www.vatican.va/roman curia/synod/documents/rc_synod_doc_20110202_lineamenta-xiii-assembly_it.html (30.05.2014).

Synod of Bishops, XIII Ordinary General Assembly, The new evangelization for the transmission of the christian faith, Instrumentum laboris (2012), http://www.vatican.va/ roman_curia/synod/documents/rc_synod_doc_20120619_instrumentum-xiii_en.html (30.05.2014).

Congregation for the Clergy, Circular letter Omnes christifideles on pastoral councils, 21.01.1973, http://www.vatican.va/roman_curia/congregations/cclergy/documents/rc con_cclergy_doc_19730125_omnes-christifideles_it.html (30.05.2014). 
Borras A., La parrocchia. Diritto canonico e prospettive pastorali, Bologna 1997.

Gręźlikowski J., Rady parafialne w świetle aktualnych potrzeb Kościoła w Polsce, „Homo Dei” 270 (2004/1), 45-58.

Lanza S., Progetto, discernimento, verifica pastorale, in: Aa. Vv., Creatività dello Spirito Santo e programmazione pastorale, Roma 1998. 